\title{
Paraneoplastic Choreoathetosis in a Patient with Small Cell Lung Carcinoma and Anti-CRMP5/CV2: A Case Report
}

\author{
Dogu Aydin ${ }^{a} \quad$ Finn Somnier ${ }^{b} \quad$ Lisbeth H. Lassen ${ }^{a}$ \\ ${ }^{a}$ Department of Neurology, Rigshospitalet Glostrup, University of Copenhagen, and \\ ${ }^{b}$ Department of Autoimmunology and Biomarkers, Statens Serum Institut, Copenhagen, \\ Denmark
}

\section{Key Words}

Paraneoplastic choreoathetosis - Anti-CRMP5/CV2 antibodies - Small cell lung carcinoma . Movement disorder $\cdot$ Cancer $\cdot$ Nervous system

\begin{abstract}
Introduction: The occurrence of more or less monosymptomatic paraneoplastic choreoathetosis associated with anti-CRMP5/CV2 antibodies is rare. Typically, such autoantibodies are associated with a more classical syndrome - paraneoplastic encephalomyelitis. Frequently, small cell lung carcinoma (SCLC) is the related neoplastic finding. Case Report: We present a 71-year-old woman who developed visual symptoms with papilledema and chorea. AntiCRMP5/CV2 antibodies were a feature of both the serum and cerebrospinal fluid. Although SCLC was suspected already at the time of the initial examinations, no signs of primary or metastatic tumors were revealed on chest X-ray, MRI or whole-body PET scan. EEG and bronchoscopy were also unremarkable. However, 6 months after the onset, a repeated PET scan and subsequent bronchoscopic biopsy revealed SCLC. In spite of chemotherapy, the SCLC progressed, and the patient died 14 months after the onset of the symptoms. Conclusion: We report paraneoplastic choreoathetosis associated with anti-CRMP5/CV2 antibodies. Such published case histories are rare. Although expected, we did not find any reduced signal intensity at the basal ganglia on the T1-weighted or increased intensity on the T2-weighted MRI scans.


Aydin et al.: Paraneoplastic Choreoathetosis in a Patient with Small Cell Lung Carcinoma and Anti-CRMP5/CV2: A Case Report

\section{Introduction}

The occurrence of anti-CRPM5/CV2 antibodies is usually associated with paraneoplastic encephalomyelitis and with chorea as a possible feature, although typically being a multifocal syndrome [1]. On the other hand, paraneoplastic choreoathetosis (PNCA) is defined as a more or less isolated movement disorder as a remote effect of a cancer on the nervous system [1]. In PNCA, the most frequent neoplastic finding is small cell lung carcinoma (SCLC) [1]. We report such a rare case with papilledema as the only additional symptom.

\section{Case Report}

A 71-year-old woman presented to a general practitioner with a 5-day history of progressively decreasing left-sided vision. The sequelae of a prior surgery for a right-sided acoustic neuroma had left her with blindness on her right eye, right-sided hearing loss and peripheral facial palsy. She was a smoker with a history of 60 pack-years, and did not use medication daily. No family disposition could be obtained, since the patient was adopted. Over the previous 6 months, there had been an unintended weight loss of $10 \mathrm{~kg}$. An ophthalmological examination revealed ischemic papillary stasis, which was treated with prednisone $80 \mathrm{mg} /$ day. Furthermore, there was a sudden onset of involuntary movements of the upper and lower limbs. She was admitted to a hospital 7 days later. On neurological examination, she was alert and orientated. In addition to the already known features related to the treatment of an acoustic neuroma, there was universal choreoathetosis. The initial workup, including brain MRI (with T1, T2 and FLAIR sequences), and routine chest X-ray were all unremarkable. A whole-body PET scan did not reveal signs of primary or metastatic tumors. Further examinations with EEG and bronchoscopy were unremarkable. The following analyses of serum and cerebrospinal fluid were normal: ANA, ANCA, anti-Hu, anti-Yo, Riantibody, amphiphysin-1 antibody and anti-Zic4. However, anti-CRMP5 (alias anti-CV2, method: line-blot from Euroimmun, Lübeck, Germany) was strongly positive in both media also indicating an occult cancer. She was then treated with immunoglobulin, which improved the choreiform movements. Unfortunately, over the following months, there was increasing weight loss, and the general condition worsened as well. Six months after the onset, a repeated PET scan showed foci suspicious of malignancy in the mediastinal lymph nodes and the right lung hilum. In May 2007, subsequent bronchoscopic biopsy revealed an SCLC. In spite of chemotherapy, repeated lung CT scans showed further progression, and the PNCA did not improve. As a palliative treatment for the SCLC, radiotherapy (10 Gy) was then given. She died 14 months after the onset of the symptoms, and her death was attributed to the SCLC complicated with infection.

\section{Discussion}

PNCA is an extremely rare disorder [1]. Most likely, it is caused by a damage of the nervous tissue by the remote effects of onconeuronal autoimmune factors including autoantibodies [1]. Associated with autoantibodies against intracellular epitopes, such a disorder is likely to be $\mathrm{T}$ cell mediated. It is provoked by cancer located outside the central nervous system and most frequently SCLC. However, based on the underlying pathogenesis, paraneoplastic disorders of the central nervous system can be divided into two categories: group 
Aydin et al.: Paraneoplastic Choreoathetosis in a Patient with Small Cell Lung Carcinoma and Anti-CRMP5/CV2: A Case Report

1 with antibodies against intracellular antigens (e.g. Hu and CRMP5/CV2) and group 2 with antibodies binding to superficial cell membrane structures (e.g. receptors and channels).

The occurrence of papilledema is explicable in terms of ischemic papillary stasis due to inflammation. Choreiform movements in the paraneoplastic syndrome have been reported in case reports of patients with different types of cancer, SCLC being the most frequently reported (table 1). Common for nearly all reported cases of PNCA due to SCLC is the presence of CRPM5/CV2 antibodies (table 1). Additionally, in patients with paraneoplastic chorea, the expected brain MRI finding is reduced signal intensity from the basal ganglia on the T1-weighted and increased intensity on the T2-weighted MRI scans. In the current case, however, such abnormalities were not observed. However, in half of the reported cases of PNCA, the MRI did not show changes in the basal ganglia (table 1). Thus, in patients who present with new-onset and rapidly developing movement disorders and no pathological findings by routine biochemistry or brain MRI, a paraneoplastic origin should be considered. Moreover, paraneoplastic symptoms may occur months before the neoplastic disorder becomes detectable. An adequate diagnosis is a primary task for a neurologist, although a prompt and intensive workup may include an interdisciplinary team (e.g. ophthalmologists, radiologists, pulmonologists and oncologists). The outcome and prognosis depend on an early establishment of the diagnosis and a prompt start of therapy.

Movement disorders, including chorea, are common in the elderly. In view of the extreme scarcity of reported PNCA in contrast to a much more common occurrence of antiCRMP5/CV2, PNCA may be underdiagnosed.

\section{Statement of Ethics}

The authors have no ethical conflicts to disclose.

\section{Disclosure Statement}

There are no conflicts of interest in relation to this publication.

\section{References}

1 Nakae Y, Ikeda S, Yamamoto R, Tanaka F, Johkura K: Hemichorea in a thymoma patient without anti-CRMP5 antibody. Neurol Sci 2014;35:629-630.

-2 Jiménez Caballero PE: Paraneoplastic chorea caused by anti-CRMP5 antibodies associated with small cell lung cancer. Neurologia 2014;29:446-447.

-3 Bohrer S, De La Villeon G, Carneiro M, Fernandez C, Garbi D, Mace L, Milh M, Guillaumont S, Echenne B, Honnorat J, Roubertie A: Acute-onset chorea, dystonia, and cardiac fibroelastoma in a child: a paraneoplastic association? Mov Disord 2013;28:250-252.

-4 Vick A, Kraemer RR, Morris JL, Willett LL, Centor RM, Estrada CA, Rodriguez JM: A 60-year-old woman with chorea and weight loss. J Gen Intern Med 2012;27:747-751.

-5 Saito H, Ohtsuka K, Takahashi H, Miura H, Taguchi T, Nanri K: A case of lung-adenocarcinoma presenting with chorea with bilateral basal ganglial lesions on MRI (in Japanese). Rinsho Shinkeigaku 2010;50:556560 .

6 Martinková J, Valkovic P, Benetin J: Paraneoplastic chorea associated with breast cancer. Mov Disord 2009;24:2296-2297.

7 Kleinig TJ, Thompson PD, Kneebone CS: Chorea, transverse myelitis, neuropathy and a distinctive MRI paraneoplastic manifestations of probable small cell lung cancer. J Clin Neurosci 2009;16:964-966.

-8 Kellinghaus C, Kraus J, Blaes F, Nabavi DG, Schäbitz WR: CRMP-5-autoantibodies in testicular cancer associated with limbic encephalitis and choreiform dyskinesias. Eur Neurol 2007;57:241-243. 
Aydin et al.: Paraneoplastic Choreoathetosis in a Patient with Small Cell Lung Carcinoma and Anti-CRMP5/CV2: A Case Report

-9 Krolak-Salmon P, Androdias G, Meyronet D, Aguera M, Honnorat J, Vighetto A: Slow evolution of cerebellar degeneration and chorea in a man with anti-Yo antibodies. Eur J Neurol 2006;13:307-308.

10 Dorban S, Gille M, Kessler R, Piéret F, Declercq I, Sindic CJ: Chorea-athetosis in the anti-Hu syndrome (in French). Rev Neurol (Paris) 2004;160:126-129.

11 Kinirons P, Fulton A, Keoghan M, Brennan P, Farrell MA, Moroney JT: Paraneoplastic limbic encephalitis (PLE) and chorea associated with CRMP-5 neuronal antibody. Neurology 2003;61:1623-1624.

12 Tan EK, Chan LL, Auchus AP, Wong MC: Reversible choreoathetosis in primary cerebral lymphoma: clinicoradiologic correlation. Eur Neurol 2003;50:53-54.

13 Vernino S, Tuite P, Adler CH, Meschia JF, Boeve BF, Boasberg P, Parisi JE, Lennon VA: Paraneoplastic chorea associated with CRMP-5 neuronal antibody and lung carcinoma. Ann Neurol 2002;51:625-630.

14 Kujawa KA, Niemi VR, Tomasi MA, Mayer NW, Cochran E, Goetz CG: Ballistic-choreic movements as the presenting feature of renal cancer. Arch Neurol 2001;58:1133-1135.

15 Tani T, Piao Y, Mori S, Ishihara N, Tanaka K, Wakabayashi K, Takahashi H: Chorea resulting from paraneoplastic striatal encephalitis. J Neurol Neurosurg Psychiatry 2000;69:512-515.

16 Nuti A, Ceravolo R, Salvetti S, Gambaccini G, Bonuccelli U, Capochiani E: Paraneoplastic choreic syndrome during non-Hodgkin's lymphoma. Mov Disord 2000;15:350-352.

Table 1. Reported cases of PNCA

\begin{tabular}{|c|c|c|c|c|c|c|}
\hline First author [Ref.], year & $\begin{array}{l}\text { Age, } \\
\text { years }\end{array}$ & Sex & Symptom & Tumor & Antibodies & $\begin{array}{l}\text { Reduced intensity } \\
\text { on T1- and incre- } \\
\text { ased intensity on } \\
\text { T2-weighted MRI }\end{array}$ \\
\hline Nakae [1], 2014 & 72 & M & hemichorea & thymoma & no anti-CRMP5 & no \\
\hline Jiménez Caballero [2], 2014 & 469 & M & chorea & SCLC & positive anti-CRMP5/CV2 & no \\
\hline Bohrer [3], 2013 & 7 & M & $\begin{array}{l}\text { choreo- } \\
\text { dystonia }\end{array}$ & cardiac & no anti-CRMP5/CV2 or anti-NMDA & no \\
\hline Vick [4], 2012 & 69 & $\mathrm{~F}$ & chorea & SCLC & positive anti-CRMP5 & yes \\
\hline Saito [5], 2009 & 63 & M & chorea & lung ACA & no anti-CRMP5 or anti-Yo & yes \\
\hline Martinkova [6], 2009 & 60 & $\mathrm{~F}$ & chorea & breast & positive anti-Hu and anti-Ri & no \\
\hline Kleinig [7], 2009 & 73 & $\mathrm{~F}$ & chorea & SCLC & no anti-Hu, anti-Ri or anti-Yo; anti-CRMP5 not tested & yes \\
\hline Kellinghaus [8], 2007 & 40 & M & chorea & testicular & positive anti-CRMP5 & no \\
\hline Krolak-Salmon [9], 2006 & 72 & M & chorea & lung ACA & positive anti-Yo & n.a. \\
\hline Dorban [10], 2004 & 48 & $\mathrm{~F}$ & chorea & SCLC & positive anti-Hu; no anti-CRMP5/CV2 & yes \\
\hline Kinirons [11], 2003 & 62 & $\mathrm{~F}$ & chorea & SCLC & positive anti-CRMP5 & yes \\
\hline $\operatorname{Tan}[12], 2003$ & 62 & $\mathrm{~F}$ & chorea & PCL & n.a. & n.a. \\
\hline Vernino [13], 2002 & 69 mean & $\begin{array}{r}10 \mathrm{~F} \\
6 \mathrm{M}\end{array}$ & chorea & $\begin{array}{l}\text { SCLC } 12 \text {, renal } 1 \text {, } \\
\text { NHL } 1 \text {, lung mass } 2\end{array}$ & positive anti-CRMP5 in all & $\begin{array}{l}\text { yes }(n=5), \text { no ( } 3) \text {, } \\
\text { n.a. (8) }\end{array}$ \\
\hline Kujawa [14], 2001 & 55 & M & chorea & renal & n.a. & no \\
\hline Tani [15], 2000 & 73 & M & chorea & SCLC & positive $68-\mathrm{kDa}$ antibody; no anti-Hu, anti-Yo and anti-Ri & yes \\
\hline Nuti [16], 2000 & 70 & M & chorea & NHL & no anti-Hu or anti-Yo; anti-CRMP5 not tested & n.a. \\
\hline
\end{tabular}

ACA = Adenocarcinoma; NHL = non-Hodgkin lymphoma; $\mathrm{PCL}=$ primary cerebral lymphoma; .a. = not assessed. 